\title{
FUNGOS PREDADORES DE NEMATOIDES EM AMOSTRAS DE SOLO DO MUNICÍPIO DE ALEGRETE-RS
}

\section{RESUMO}

${ }^{1}$ Mestranda do Programa de Pós-Graduação em Veterinária/UFPEL; ${ }^{2}$ Acadêmica da Faculdade de Veterinária/UFPEL; ${ }^{3}$ Mestrando do Programa de Pós-Graduação em Zootecnia/UFPEL; ${ }^{4}$ Doutoranda do programa de Pós-Graduação em Veterinária/UFPEL; ${ }^{5}$ Pós-Doutoranda do Programa de Pós-Graduação em Veterinária/UFPEL; ${ }^{6}$ Professora, Doutora, Departamento de Veterinária Preventiva, Faculdade de Veterinária/UFPEL; ${ }^{7}$ Professor, Doutor, Departamento de Veterinária Preventiva, Faculdade de Veterinária/UFPEL.

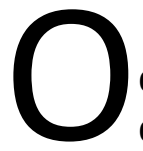

controle das endoparasitoses representa um grande desafio em vista do desenvolvimento de resistência dos parasitas aos medicamentos anti-helmínticos disponíveis, o que estimula a busca por formas alternativas de controle. Os fungos nematófagos são espécies capazes de utilizar larvas de nematoides como forma de nutrição podendo ser classificados como ovicidas, endoparasitos ou predadores. Fungos predadores são telúricos, saprofíticos e, em condições especiais, produzem armadilhas tridimensionais para capturar os nematoides. Por essa atividade nematofágica, os fungos predadores podem ser utilizados como controladores biológicos de larvas de parasitas no meio ambiente, evitando a ingestão dessas larvas pelos animais e sua posterior infestação. Este trabalho objetivou o isolamento de fungos predadores de nematoides a partir de amostras de solo do município de Alegrete-RS. Para isolar os fungos utilizou-se a técnica de espalhamento do solo sobre ágar-água a $2 \%$, adicionando-se nematoides Panagrellus redivivus como isca para demonstração das estruturas predatórias fúngicas. Mediante aprisionamentos coletou-se o micélio reprodutivo fúngico que foi ressemeado em meio de cultura PDA para purificação das colônias. A identificação dos isolados foi realizada visualizando-se as características morfológicas das colônias, microcultura e exame direto com base em chaves classificatórias. Foram isolados, a partir das amostras de solo, duas espécies de fungos predadores identificados como Fusarium solani e Fusarium xylarioides. Portanto, conclui-se que existem fungos telúricos com atividade nematofágica no município de Alegrete-RS com uso potencial para descontaminação de pastagens.

Palavras-chave: Controle biológico. Fusarium. Parasitas gastrintestinais. Fungos nematófagos. Desinfestação de pastagens. 


\section{INTRODUÇÃO}

O controle das parasitoses gastrintestinais é um tema de grande relevância para a medicina veterinária e que envolve aspectos econômicos, sanitários e ambientais. Para o setor agropecuário, as endoparasitoses são sinônimo de perdas econômicas, pois elevam os custos da produção diretamente nos gastos com medicamentos anti-helmínticos e indiretamente com a redução no ganho de peso dos animais em decorrência da espoliação realizada pelos parasitas (STOTZER et al., 2014).

A ingestão da pastagem contaminada por larvas de parasitas em estágio infectante é a principal forma de infestação dos animais de produção. Pastagens densamente infestadas acabam inviabilizando a criação nesses locais, especialmente de espécies que apresentam baixa resiliência às helmintíases gastrintestinais como os ovinos (AMARANTE; SALES, 2007).

Estima-se que 95\% das formas parasitárias estejam no ambiente sob a forma de ínstares imaturos enquanto apenas os $5 \%$ restantes estão na forma de parasitas adultos infestando os hospedeiros (LABRUNA, 2004). As larvas de helmintos em estágio infectante podem permanecer viáveis nas pastagens por um período de até três meses mesmo na ausência de animais. Em estações chuvosas há o aumento da quantidade de larvas disponíveis na pastagem (SOUZA et al., 2000).

Os medicamentos anti-helmínticos foram desenvolvidos para atuarem apenas no interior do hospedeiro. A eliminação dos resíduos desses fármacos através das fezes e urina dos animais também ocasiona transtornos ao meio ambiente como a destruição da fauna coprófaga responsável pela degradação do bolo fecal e enriquecimento do solo (GILAVERTE et al., 2012).

Em decorrência da alta suscetibilidade dos animais aos parasitas, falta de orientação técnica dos produtores e esquemas profiláticos inapropriados ocorreu a seleção de parasitas altamente resistentes aos princípios ativos disponíveis para o tratamento das parasitoses (RAMOS et al., 2002). Essa resistência, relatada em diversas espécies de animais de produção (AHID et al., 2008; HOLSBACK et al., 2015; VERA, 2014), estimula a pesquisa por alternativas para o controle das helmintíases gastrintestinais. 
O controle biológico é um método alternativo de controle não químico que consiste na utilização de predadores antagonistas objetivando a redução de uma população que esteja ocasionando prejuízos econômicos (MOTA et al., 2003).

Os fungos nematófagos são espécies telúricas que possuem a capacidade de utilizar larvas de nematoides em diferentes estágios de desenvolvimento como forma de nutrição podendo ser classificados de acordo com seu mecanismo de ação como: ovicidas, endoparasitos ou predadores. Os ovicidas também chamados oportunistas destroem os ovos de parasitas penetrando a membrana externa, emitindo suas hifas para o interior do ovo e consumindo o embrião (DE FREITAS, 2010). As espécies de fungos endoparasitos são parasitas obrigatórios e infectam os nematoides quando esses ingerem seus esporos ou pela adesão dos conídios à cutícula do helminto com posterior desenvolvimento do tubo germinativo para penetração na cutícula e destruição interna do nematoide (CARNEIRO, 1992). Já os fungos nematófagos predadores são espécies saprofíticas que, em condições de restrição nutricional, desenvolvem estruturas tridimensionais na forma de anéis constritores, anéis não constritores, redes, hifas adesivas e botões adesivos que servem para capturar os nematoides. Após a captura da larva o fungo predador emite suas hifas para o interior do nematoide nutrindo-se dele (MOTA et al., 2003).

Os fungos nematófagos causam a interrupção no ciclo de vida dos parasitas atuando em suas formas de vida livre promovendo consequentemente a descontaminação das pastagens pela supressão do número de larvas disponíveis no ambiente (LARSEN, 1999).

O objetivo do presente trabalho foi pesquisar a presença de fungos predadores de nematoides em amostras de solo provenientes do município de Alegrete, estado do Rio Grande do Sul.

\section{MATERIAL E MÉTODOS}

\section{Cultura de nematoides}

Para estimular o desenvolvimento das estruturas predatórias pelos fungos nematófagos foram utilizados nematoides de vida livre Panagrellus redivivus. A cultura pura dos 
nematoides foi cedida pelo Centro de Diagnóstico e Pesquisa em Micologia Veterinária (MICVET), da Universidade Federal de Pelotas (UFPEL).

\section{Obtenção das amostras de solo}

Foi coletado, de forma aleatória, um total de dez amostras de solo no município de AlegreteRS, contendo aproximadamente 100 gramas cada. Cinco amostras eram provenientes de áreas com constante circulação de animais, e as outras cinco foram coletadas em locais restritos ao acesso de animais. Todas as coletas foram efetuadas a partir da camada mais superficial do solo (rizosfera) onde se concentra a matéria orgânica. No momento da coleta foram descartadas pedras e resíduos vegetais. As amostras foram acondicionadas em caixas isotérmicas e encaminhadas ao laboratório de micologia (MICVET) para pesquisa de fungos com atividade nematicida.

\section{Processamento das amostras em laboratório}

As amostras de solo foram homogeneizadas e processadas em triplicata pela técnica de espalhamento do solo de Duddington (1950) modificada por Santos (1991) que consiste em semear dois gramas da amostra de solo em formato de cruz na placa de Petri contendo meio de cultura ágar-água a $2 \%$. Ao conjunto adicionaram-se nematoides $P$. redivivus para estimular o fungo a produzir as estruturas predatórias. As placas semeadas foram incubadas em estufa BOD a $25{ }^{\circ} \mathrm{C}$ por 24 horas. Após esse período as placas foram visualizadas diariamente ao microscópio óptico por até dez dias para avaliar o desenvolvimento de estruturas predatórias e de aprisionamentos de $P$. redivivus.

\section{Purificação das colônias fúngicas}

Ao identificar-se o aprisionamento de nematoides na placa de Petri, o micélio aéreo (reprodutivo) do fungo foi coletado com uma alça de platina e transferido para uma placa de Petri contendo meio de cultura PDA (Potato Dextrose Agar), que foi incubada em estufa BOD a $25{ }^{\circ} \mathrm{C}$ para desenvolvimento e purificação das colônias fúngicas.

\section{Confirmação da atividade predatória}

Para confirmar a atividade predatória dos fungos isolados foram obtidos discos de cinco milímetros de diâmetro a partir dos bordos das colônias purificadas e dispostos sobre meio 
de cultivo PDA, adicionou-se nematoides $P$. redivivus e o conjunto foi incubado em estufa BOD a $25{ }^{\circ} \mathrm{C}$ para confirmar o desenvolvimento dos órgãos de captura.

\section{Identificação de fungos predadores}

A identificação dos fungos isolados foi realizada por exame direto das colônias e técnica de microcultura em lâmina. Para o exame direto foram coletados fragmentos das colônias fúngicas com uma alça de platina que foram dispostos entre lâmina e lamínula e corados com Lactofenol (Azul de algodão).

Na técnica de microcultura em lâmina ou microcultivo um quadrado de ágar PDA de aproximadamente dois $\mathrm{cm}^{2}$ foi depositado sobre uma lâmina histológica. O fungo de interesse foi semeado nos quatro cantos do quadrado de ágar e uma lamínula foi colocada sobre a semeadura. Na sequência o conjunto (lâmina e lamínula) foi colocado em uma placa de Petri sobre um papel filtro e um suporte. O papel filtro foi umedecido com água esterilizada e a placa de Petri fechada incubada a $25{ }^{\circ} \mathrm{C}$ até o crescimento fúngico (RIDELL, 1950).

A visualização das estruturas fúngicas (hifas, conidióforos, macroconídios, microconídios, clamidoconídios), do exame direto e da microcultura em lâmina foi realizada através de microscópio óptico de acordo com características morfotinturiais, tendo como base chaves classificatórias (BOOTH, 1971; LACAZ et al., 1998).

\section{RESULTADOS E DISCUSSÃO}

Após 48 horas de incubação das placas contendo as amostras de solo, observaram-se os aprisionamentos de $P$. redivivus com formação de redes tridimensionais como instrumento de captura em duas das dez amostras. As duas amostras positivas foram identificadas como A e B. Os micélios reprodutivos dos fungos contidos nas amostras A (Figura 1A) e B (Figura 1B) diferiram morfologicamente na observação ao microscópio óptico, indicando a possibilidade de se tratarem de espécies distintas. 


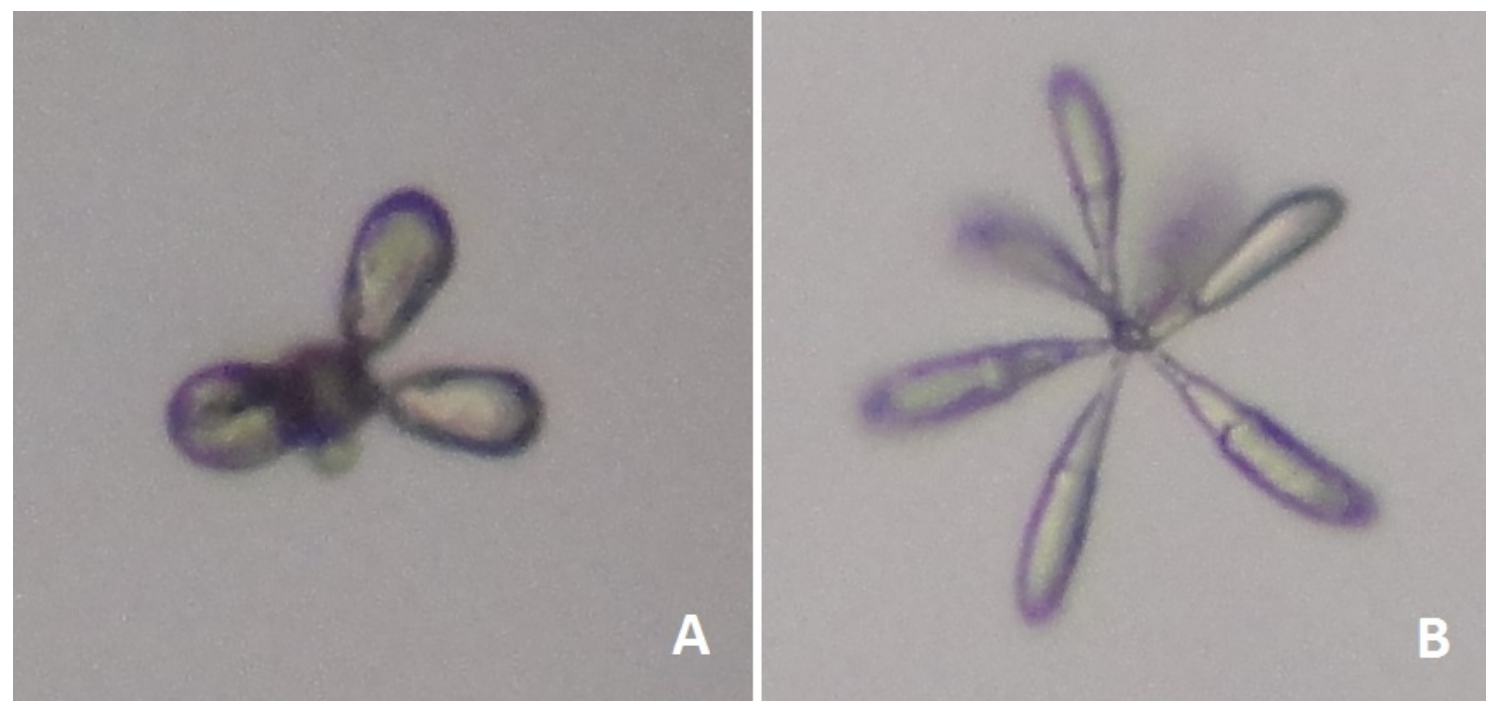

Figura 1 - Morfologia dos conidióforos durante o aprisionamento nas amostras A e B (400X).

A partir dos micélios reprodutivos das duas amostras analisadas foram isolados, em meio de cultura PDA, dois fungos predadores com atividade nematofágica, macro e micromorfologicamente distintos, ambos pertencentes ao gênero Fusarium.

A espécie proveniente da amostra A foi identificada como Fusarium solani e apresentou uma colônia de rápido crescimento em meio de cultivo PDA (mais de dois centímetros de diâmetro em sete dias de incubação) de coloração branca a bege e aspecto cremoso (Figura 2A). Na microscopia, F. solani apresentou macroconídios levemente curvados curtos com um a três septos, clamidoconídios abundantes na posição apical (Figura 2B) e em cadeia (Figura 2C), além de numerosos microconídios (Figura 3D). 


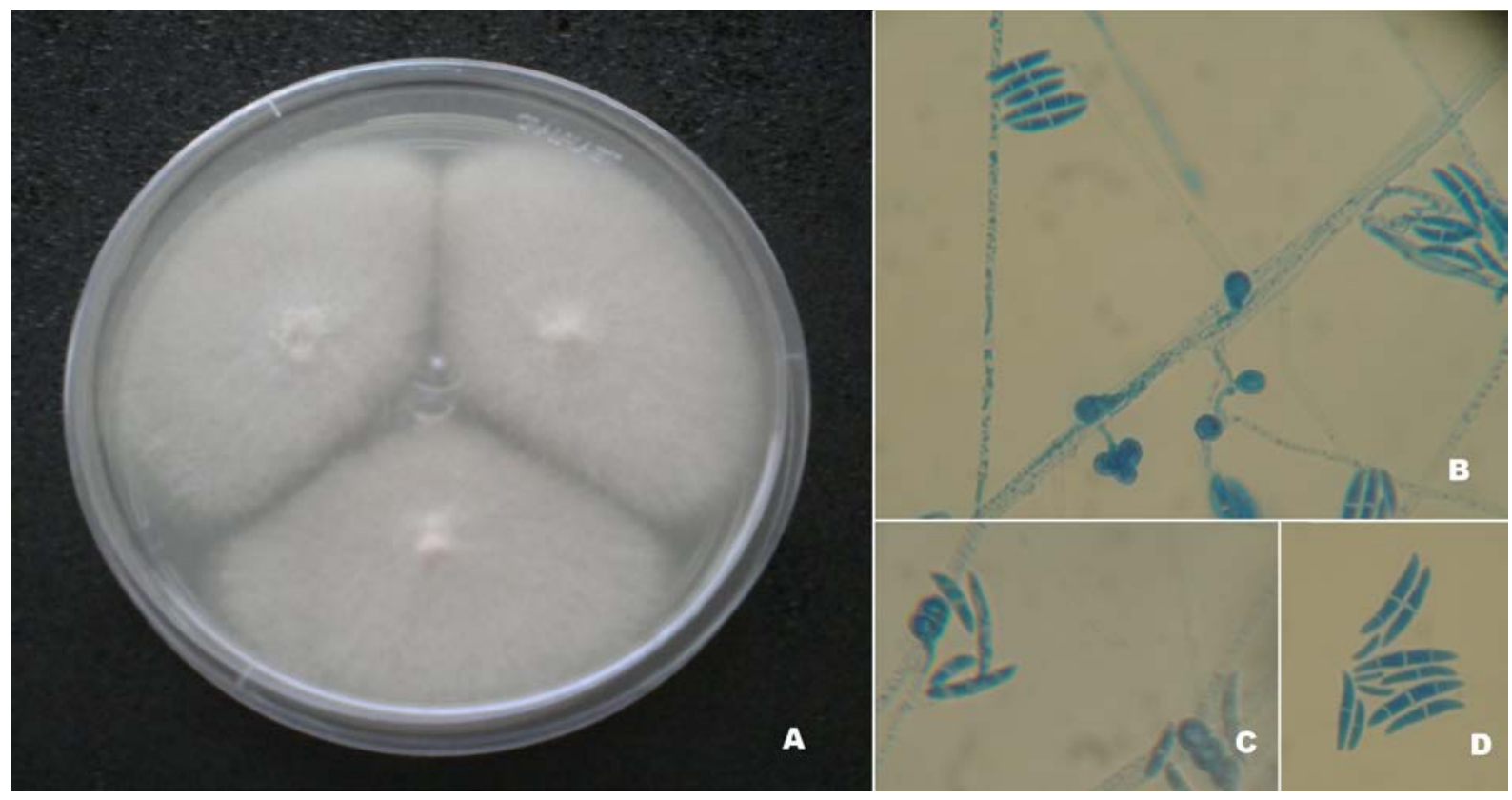

Figura 2 - Aspecto macroscópico e microscópico de Fusarium solani (400X).

Em relação ao aspecto macroscópico, Fusarium xylarioides, fungo isolado da amostra B, também apresentou crescimento rápido em PDA, de coloração inicialmente branca com reverso rósea tornando-se avermelhada com o amadurecimento da colônia, aspecto algodonoso e micélio aéreo alto e denso (Figura 3A). Microscopicamente observou-se ausência de microconídios, macroconídios com até cinco septos com célula apical em formato de gancho (Figura 3B), clamidoconídios raros, apicais (Figura 3C) e intercalares (Figura 3D). 


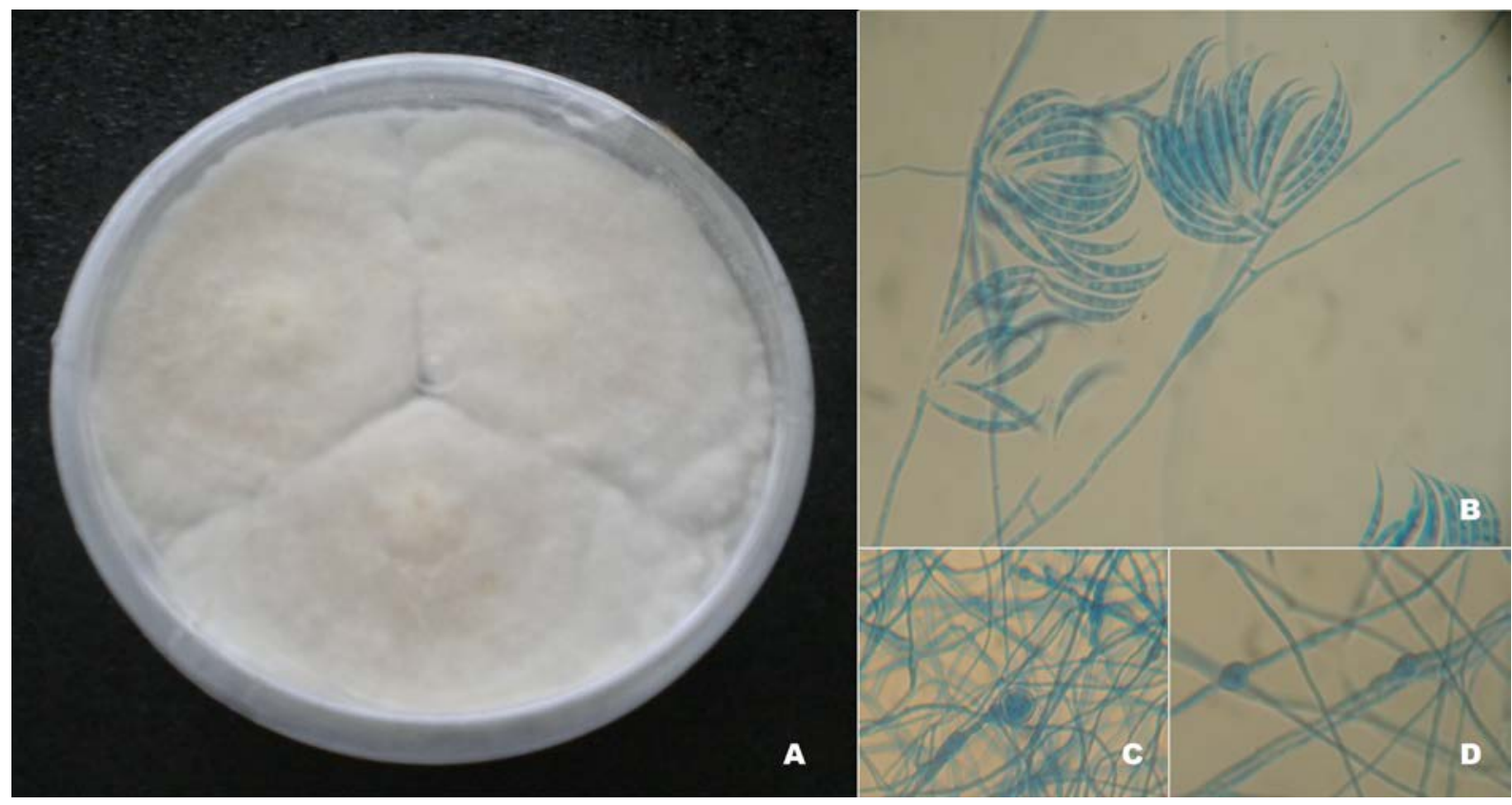

Figura 3 - Aspecto macroscópico e microscópico de Fusarium xylarioides (400X).

Ao compararem-se as estruturas reprodutivas demonstradas no ato de aprisionamento (Figura 1) e na cultura purificada dessas estruturas (Figuras 2D e 3B) são evidentes as diferenças morfológicas entre conidióforos e conídios tanto com relação à forma quanto relacionado ao tamanho dos conídios como descrito por Cooke e Godfrey (1964). Segundo SANTOS et al. (1991), os conídios das culturas que contém nematoides são maiores quando comparados aos conídios das culturas purificadas em consequência do aporte nutricional ser mais apropriado para o crescimento do fungo predador.

Os dois fungos isolados no presente estudo demonstraram as estruturas predatórias na presença de $P$. redivivus após a purificação das colônias em meio de cultivo PDA. Essas estruturas não são visualizadas normalmente em culturas puras na ausência de nematoides (COOKE; GODFREY, 1964). A confirmação da atividade predatória é uma etapa fundamental no processo de isolamento dos fungos predadores, pois, muitas vezes, os aprisionamentos visualizados já contêm nematoides mortos ou consumidos, dessa forma, se faz necessário descartar a possibilidade do fungo isolado estar atuando apenas como decompositor ou necrófago. 
As amostras com isolamento positivo de fungos nematófagos neste estudo foram coletadas em locais sombreados. Segundo Costa (2011), a exposição à radiação ultravioleta-B, em condições de campo, pode interferir de forma negativa na germinação de conídios dos fungos controladores de fitopatógenos, Clonostachys rosea e Trichoderma spp.

O uso de fungos nematófagos não substitui ou elimina a necessidade de utilização de um controle anti-helmíntico químico nos animais. O biocontrole ocorre no ambiente, é uma prevenção à infestação, portanto, não deve ser considerado como um tratamento. $\mathrm{O}$ controle sanitário deve ser realizado de forma responsável, com orientação e critério principalmente quando o ambiente desfavorecer o desenvolvimento das formas de vida livre dos parasitas no ambiente (PADILHA; MENDOZA-GIVES, 1996).

Testes in vitro demonstraram que os princípios ativos utilizados no controle das helmintíases gastrintestinais podem inibir o crescimento de fungos nematófagos (VIEIRA, 2012). Portanto, os resíduos de fármacos anti-helmínticos eliminados pela urina e pelas fezes dos animais (GILAVERTE, 2012) podem prejudicar o desenvolvimento fúngico no ambiente afetando negativamente a eficiência de fungos biocontroladores.

A utilização de fungos nematófagos no biocontrole das endoparasitoses dos animais domésticos, apesar de promissora, possui um grande entrave, que são as formas de administração. Estudos já foram conduzidos utilizando-se cultivo de fungos nematófagos em grãos (LARSEN et al., 1995), incorporação de clamidoconídios em produtos minerais (WALLER; FAEDO,1996) ou dispositivos intra-ruminais (WALLER et al., 2001). Porém essas formulações ainda são limitadas por fatores como produção de fungos nematófagos em larga escala e controle de qualidade (ARAÚJO et al. 2004).

A pesquisa por fungos nematófagos autóctones é importante uma vez que esses fungos atuam no ambiente (ARAÚJO et al., 2004), assim sendo, estão expostos a condições ambientais que diferem para cada região. É possível que estes fungos estejam melhor adaptados às condições ambientais dos locais de onde foram coletados. Dessa forma, estudos com fungos nematófagos autóctones que levem em consideração os fatores bióticos e abióticos a que esses agentes estão submetidos em seus ambientes de origem podem 
contribuir para melhoria na utilização dos mesmos como biocontroladores de parasitas nas pastagens.

\section{CONCLUSÃO}

Conclui-se que existem fungos com atividade nematofágica no solo do município de Alegrete-RS com potencial para utilização na descontaminação de pastagens. Salienta-se a importância do planejamento de um controle parasitário integrado visando tanto o controle da infestação dos animais quanto à preservação dos fungos nematófagos no ambiente.

\section{NEMATODE-TRAPPING FUNGI IN SOIL SAMPLES FROM ALEGRETE-RS}

\section{ABSTRACT}

E ndoparasitoses' control represents a major challenge due to the development of parasite resistance to the available anthelmintic drugs. This stimulates the search for alternative forms of control. The nematophagous fungi are able to use nematode larvae as a way of nutrition and can be classified as: ovicides, endoparasites or nematodetrapping fungi. The nematode-trapping fungi are terrestrial, saprophytic, and in special conditions, produce three-dimensional traps to capture the nematodes. For this predatory activity fungi can be used as biological controllers of parasite larvae in the environment preventing their ingestion by the animals and their subsequent infestation. This work aimed to isolate nematode-trapping fungi from soil samples from the city of Alegrete-RS. The soil sprinkling technique was used to isolate fungi from the soil samples. It consists on adding Panagrellus redivivus nematodes to a $2 \%$ agar-water plate as bait for the demonstration of the fungal predatory structures. By imprisonment, the fungal reproductive mycelium was collected and put in PDA culture medium for the purification of the colonies. The identification of the isolates was performed by viewing the morphological characteristics of the colonies, microculture and direct examination based on qualifying keys. Two species of nematode-trapping fungi were isolated from the soil samples and identified as Fusarium solani and Fusarium xylarioides. Therefore, it is concluded that there are terrestrial fungi with nematicide activity in the soil of Alegrete-RS with potential use for the decontamination of pastures.

Keywords: Biological control. Fusarium. Gastrointestinal parasites. Nematophagous fungi. Decontamination of pastures. 


\section{HONGOS DEPREDADORES DE NEMÁTODOS EN LAS MUESTRAS DE SUELO DEL MUNICIPIO DE ALEGRETE-RS}

\section{RESUMEN}

$\mathrm{E}$ control de endoparásitos representa un gran desafío en vista del desarrollo de resistencia de los parásitos a los fármacos antihelmínticos disponibles, lo que estimula la búsqueda de formas alternativas de control. Las especies de hongos nematófagos son capaces de utilizar las larvas de nematodos como una forma de nutrición por lo tanto pueden ser clasificados como ovicidas, endoparásitos o depredadores. Los hongos son depredadores telúricos, saprófitos y en condiciones especiales, producen trampas tridimensionales para capturar los nematodos. Para esta actividad nematofágica los hongos depredadores pueden utilizarse como controladores biológicos de las larvas de parásitos en el medio ambiente evitando la ingesta de las larvas por los animales y posterior infestación. Este estudio tuvo como objetivo aislar hongos nemátodos depredadores de muestras de suelo del municipio de Alegrete. Para aislar los hongos se utilizó la técnica de dispersión de suelo en Agar-agua al 2\%, añadiendo nematodos Panagrellus redivivus como cebo para la demostración de las estructuras de hongos depredadores. Mediante prensión se colectó el micelio reproductivo fúngico que fue resembrado en medio de cultivo PDA para la purificación de las colonias. La identificación de los aislamientos se realizó mediante la visualización de las características morfológicas de las colonias, microcultivo y el examen directo basado en claves de clasificación. Fueron aisladas de dos muestras de suelo, dos especies de hongos depredadores identificados como Fusarium solani y Fusarium xylarioides. Por lo tanto, se concluye que hay hongos telúricos con actividad nematofágica en el municipio de Alegrete-RS con uso potencial para la descontaminación de la pastura.

Palabras clave: Control biológico. Fusarium. Parásitos gastrointestinales. Hongos depredadores. Desinfección de pastos.

\section{AGRADECIMENTOS}

Os autores agradecem à Universidade Federal de Pelotas pelas instalações onde o presente trabalho foi desenvolvido e ao CNPq pela concessão das bolsas de mestrado e de iniciação científica que propiciaram o desenvolvimento dessa pesquisa. 


\section{REFERÊNCIAS}

AHID, S. M. M.; BEZERRA, A. C. D. S.; SOARES, H. S.; et al. Diagnóstico da resistência dos nematóides gastrintestinais a anti-helmínticos em rebanhos caprino e ovino do RN. Acta Veterinaria Brasilica, v. 2, n. 1, p. 16-19, 2008.

AMARANTE, A. F. T.; SALES, R. O. Controle de Endoparasitoses dos Ovinos: Uma Revisão. Revista Brasileira de Higiene e Sanidade Animal, v. 1, n. 2, p. 14-36, 2007.

ARAÚJO, J. V.; MOTA, M. A.; CAMPOS, A. K. Controle biológico de helmintos parasitos de animais por fungos nematófagos. Revista Brasileira de Parasitologia Veterinária, v. 13, n. 1, p. 165-170, 2004.

BOOTH, C. The Genus Fusarium. Kew: Commonwealth Agricultural Bureau. Commonwealth Mycological Institute, 1971. 237p.

CARNEIRO, R. M. G. Princípios e tendências do controle biológico de nematóides com fungos nematófagos. Pesquisa Agropecuária Brasileira, v. 27, p. 113-121, 1992.

COOKE, R. C.; GODFREY, B. E. S. A key of nematode destroying fungi. Transactions British Mycological Society, Cambridge, v. 47, p. 61-74, 1964.

COSTA, L. B. Efeito da radiação ultravioleta-B sobre Trichoderma spp. e Clonostachys rosea, agentes de biocontrole de fitopatógenos. Lavras: UFLA, 2011. 80p. Dissertação (Mestrado em Fitopatologia), Faculdade de Agronomia, Universidade Federal de Lavras, 2011.

DE FREITAS, L. G. Destruição de ovos de Toxocara canis pelo fungo nematófago Pochonia chlamydosporia. Revista da Sociedade Brasileira de Medicina Tropical, v. 43, n. 1, p. 102104, 2010.

DUDDINGTON, C. L. A new predacious species of Trichotecium. Transactions of the British Mycological Society, v. 32, p. 284-287, 1950.

GILAVERTE, S.; MONTEIRO, A. L. G.; FERNANDES, M. A. M.; et al. Fauna edáfica e impacto ambiental do resíduo de moxidectina presente nas fezes de ruminantes. Current Agricultural Science and Technology, v. 18, n. 3, p. 233-243, 2012.

HOLSBACK, L.; DA SILVA, M. A.; PATELLI, T. H. C.; et al. Resistência de Haemonchus, Cooperia, Trichostrongylus e Oesophagostomum à ivermectina em bovinos de leite no Paraná. Semina: Ciências Agrárias, n. 36, v. 3, p. 2031-2036, 2015.

LABRUNA, M. B. Biologica-ecologia de Rhipicephalus sanguineus (Acari: Ixodidae). Revista Brasileira de Parasitologia Veterinária, v. 13, Supl. 1, p. 123-124, 2004. 
LACAZ, C. S.; PORTO, E.; HEINS-VACCARI, E. M.; MELO, N. T. Guia para identificação: fungos, actinomicetos e algas de interesse médico. São Paulo: Sarvier, 1998.

LARSEN, M. Biological control of helminths. International Journal for Parasitology, v. 29, n. 1, p. 139-146, 1999.

LARSEN, M.; NANSEN, P.; WOLSTRUP, J.; et al. Biological control of trichostrongylosis in grazing calves by means of the fungus Duddingtonia flagrans. Veterinary Parasitology, v. 60, p. 321-330, 1995.

MOTA, M. D. A.; CAMPOS, A. K.; ARAÚJO, J. V. D. Controle biológico de helmintos parasitos de animais: estágio atual e perspectivas futuras. Pesquisa Veterinária Brasileira, v. 23, n. 3, p. 93-100, 2003.

PADILHA, T.; MENDOZA-GIVES, P. Controle microbiano de formas de vida livre dos nematódeos trichostrongilídeos: uma alternativa para a higienização das pastagens. Controle dos nematódeos gastrintestinais em ruminantes. Coronel Pacheco: EMBRAPACNPGL, p. 215-235, 1996.

RAMOS, C. I.; BELLATO, V.; ÁVILA, V. S. Resistência de parasitos gastrintestinais de ovinos a alguns anti-helmínticos no Estado de Santa Catarina, Brasil. Ciência Rural, v. 32, n. 3, p. 473477, 2002.

RIDDELL, R. W. Permanent stained mycological preparations obtained by slide culture. Mycologia, v. 42, n. 2, p. 265-270, 1950.

SANTOS, M. A. Detecção, isolamento e avaliação do potencial antagonista de fungos nematófagos presentes em solos brasileiros. Viçosa: UFV, 1991. 97p. Dissertação (Mestrado em Fitopatologia), Universidade Federal de Viçosa, 1991.

SANTOS, M. A.; FERRAZ, S.; MUCHOVEJ, J. J. Detection and ecology of nematophagous fungi from Brazilian soils. Nematologia Brasileira (Brazil), v. 15, p. 121-134, 1991.

SOUZA, P. D.; BELLATO, V.; SARTOR, A. A.; et al. Período para desinfestação das pastagens por larvas de nematoides gastrintestinais de ovinos, em condições naturais nos campos de Lages, SC. Revista Brasileira de Parasitologia Veterinária, v. 9, n. 2, p. 159-164, 2000.

STOTZER, E. S.; LOPES, L. B.; ECKSTEIN, C.; et al. Impacto econômico das doenças parasitárias na pecuária. Revista Brasileira de Higiene e Sanidade Animal, v. 8, n. 3, 2014.

VERA, J. H. S. Resistência anti-helmíntica em equinos na Região Oeste do Estado de São Paulo. Ilha Solteira: UNESP, 2014. 65p. Dissertação (Mestrado em Produção Animal), Faculdade de Engenharia, Universidade Estadual Paulista Júlio de Mesquita Filho, 2014. 
VIEIRA, J. N. Suscetibilidade de fungos nematófagos a fármacos antiparasitários. Pelotas: UFPEL, 2012. 50p. Dissertação (Mestrado em Parasitologia), Programa de Pós-Graduação em Parasitologia, Universidade Federal de Pelotas, 2012.

WALLER, P. J.; FAEDO, M. The prospects for biological control of the free-living stages of nematode parasites of livestock. International Journal for Parasitology, v. 26, n. 8-9, p. $915-$ 925, 1996.

WALLER, P. J.; FAEDO, M.; ELLIS, K. The potential of nematophagous fungi to control the free-living stages of nematode parasites of sheep: towards the development of a fungal controlled release device. Veterinary Parasitology, v. 102, n. 4, p. 299-308, 2001.

Autor para correspondência: Emanoele Figueiredo Serra. Centro de Diagnóstico e Pesquisa em Micologia Veterinária/MICVET, Faculdade de Veterinária, Campus Universitário, S/N, CEP-96160-000. Capão do Leão (RS), Brasil. emanoele.serra@gmail.com 